Appl i cat i on of the compl ex beam par amet er to the desi gn of a quasi opt i cal transmi ssi on I i ne for a submill i met re wave gyr ot ron

\begin{tabular}{|l|l|}
\hline 著者 & OGAWA I, SAKAl A, I DEHARA T, KASPAREK W \\
\hline $\begin{array}{l}\mathrm{j} \text { our nal or } \\
\text { publ i cat i on t i t l e }\end{array}$ & I nt er nat i onal J our nal of El ect r oni cs \\
\hline vol une & 86 \\
\hline number & 9 \\
\hline page $\mathrm{r}$ ange & $1071-1084$ \\
\hline year & $1999-09$ \\
\hline URL & ht t p: //hdl . handl e. net /10098/1519 \\
\hline
\end{tabular}




\title{
Application of the complex beam parameter to the design of a quasi- optical transmission lime for a submillimetre wave gyrotron
}

\author{
I. OGAWA , A. SAKAI , T. IDEHARA and W. KASPAREK
}

The complex beam parameter can be used to facilitate the design of a quasi-optical transmission line which converts the output of a submillimetre wave gyrotron into a well collimated, linearly polarized beam. The quality of the beam produced by the quasi-optical antenna is improved further by removing any sidelobes. Only a small fraction of the beam power is sacrificed.

\section{Introduction}

Electromagnetic waves in the submilimetre wavelength range have applications in many fields including spectroscopy, the measurement of material properties and plasma diagnostics. Until recently, molecular vapour lasers have been the only power sources at these wavelengths (for example, Semet et al. (1980), Kawahata et al. (1988)). Like plasma scattering measurement and Thomson scattering, some applications need more intense waves (Fekete et al. 1994, Suvorov et al. 1997). High frequency gyrotrons have clear advantages at submillimetre wavelengths because of their capacity to deliver high powers (Hong et al. 1992, Idehara et al. 1992, 1993).

Before these gyrotrons can be used as power sources, it is necessary to convert the output into a well collimated, linearly polarized beam. Unlike molecular vapour lasers, gyrotrons produce hollow cone-shaped beams, which are far from what is usually required.

We have proposed the use of a quasi-optical transmission line to convert the output into a high quality beam (Ogawa et al. 1997). The system consists of a quasioptical antenna and a series of mirrors, including an elliptical mirror for beam collimation. The quasi-optical antenna converts the gyrotron output into a linearly polarized beam. This beam has sidelobes which are truncated by setting the size of an elliptical mirror located far from the antenna. The resulting high-quality beam is obtained at the expense of some power loss. The loss is small encugh to be acceptable in the low-power case, unlike high-power gyrotron applications.

In our design, the Huygens equation can be used to calculate the intensity profile of the beam produced at any point along its path.

However, if Gaussian optics can be applied, because of the long distance between the quasi-optical antenna and the elliptical mirror, the calculation time for the design of an optimized system can be significantly reduced. The main purpose of this paper is to demonstrate the effectiveness of this approach. 


\section{Spreading of beam produced by innage source}

\subsection{Descripition of the image source}

The first element in the transmission line is the quasi-optical antenna consisting of a circular waveguide with a step-cut and a parabolic reflector (see figure 1) (Vlasov and Orlova 1974). The quasi-optical antenna converts the gyrotron output into a linearly polarized beam with polarization in the $x$-direction. The beam produced has a rectangular cross-section (length $D_{\mathrm{H}}$ and width $D_{\mathrm{E}}$ ) and propagates in the direction $(0, \cos \alpha, \sin \alpha)$, where $\alpha$ is the emission angle. These values are determined by the following equations

$$
\begin{aligned}
\alpha & =\sin ^{-1} \frac{\lambda \xi_{\mathrm{mn}}^{\prime}}{2 \pi a_{\mathrm{w}}} \\
D_{\mathrm{E}} & =4 f_{\mathrm{p}} \\
D_{\mathrm{HH}} & =4 a_{\mathrm{w}} \cos \alpha
\end{aligned}
$$

where $f_{\mathrm{p}}$ is the focal length of the parabolic cylinder, $a_{\mathrm{w}}$ the waveguide radius, $\xi_{\mathrm{mn}}^{\prime}$ the $n$th root of $J_{\mathrm{in}}^{\prime}(\xi)=0$, and $\lambda$ the wavelength.

Radiation reffected from the parabolic reflector of the quasi-optical antenna is treated as if it comes from a plane image source lying behind the parabolic reflector with emission angle $\alpha$. The fields over this image source for a $\mathrm{TE}_{0 \mathrm{n}}$ mode, calculated using geometrical optics (Wada and Nakajima 1986, Brand et al. 1990), are

$$
\begin{aligned}
& E_{x}=\left(1+\frac{x^{2}}{4 f_{\mathrm{p}}^{2}}\right)^{-1 / 2} \exp (-j k y \cos \alpha) \\
& E_{y}=0 \\
& E_{z}=0 \\
& X_{x}=0 \\
& H_{y}=\frac{E_{x}}{\eta} \sin \alpha \\
& H_{z}=-\frac{E_{x}}{\eta} \cos \alpha
\end{aligned}
$$

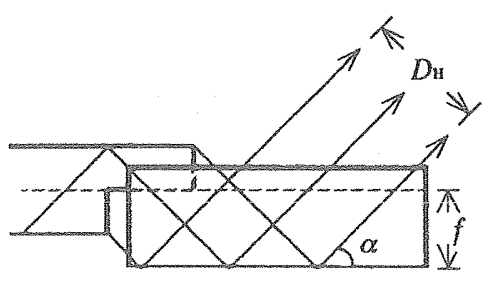

$z$

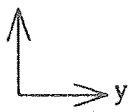

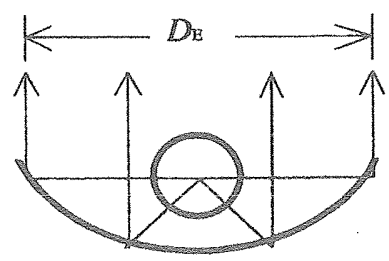

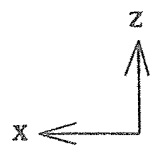

Figure 1. Quasi-optical antenna. 
in the region $-2 f_{\mathrm{p}}<x<2 f_{\mathrm{p}},-2 a_{\mathrm{w}} \cot \alpha<y<2 a_{\mathrm{w}} \cot \alpha$, and zero elsewhere. $\eta$ is the wave impedance. The origin $(0,0,0)$ is set at the centre of the image source. The fields for a $T E_{1 n}$ mode are obtained by multiplying $E_{x}$ in (4) by $\cos \phi$ because it varies with $\cos \phi$, where $\phi$ is an azimuthal angle. It follows that, for this case, we can use equation (4) if we replace $E_{x}$ by

$$
E_{x}=\left(1+\frac{x^{2}}{4 f_{\mathrm{p}}^{2}}\right)^{-3 / 2}\left(1-\frac{x^{2}}{4 f_{\mathrm{p}}^{2}}\right) \exp (-j k y \cos \alpha) .
$$

When the Poynting vector at the image source is calculated, it is easy to see that in both cases these fields describe a beam propagating in the direction $(0, \cos \alpha, \sin \alpha)$ with a rectangular cross-section.

\subsection{Fields at a distance point}

The intensity profiles of the beam are obtained by the boundary value problem of the Helmholtz equation for the image source. Its solution is equivalent to the calculation of the Huygens equation, which can be written as

$$
\psi_{\mathrm{P}}=\frac{\mathrm{j} k}{4 \pi} \iint_{\mathrm{A}} \psi_{\mathrm{S}} \frac{\exp (-j k R)}{R}(\hat{\mathrm{r}}+\hat{\mathbf{s}}) \cdot \hat{\mathrm{m}} \mathrm{d} s
$$

where $\mathrm{A}$ is the plane image source region, $R$ the distance between a source point $S$ on $A$ and the field point $P, \hat{r}$ the unit vector from point $S$ on $A$ to $P, \hat{n}$ the normal vector to $A$ and $\hat{s}$ the unit vector in the direction of the Poynting vector at the source point. If a field quantity $\psi_{\mathrm{S}}$ is given at all points on $\mathrm{A}$, then $\psi_{\mathrm{P}}$ at any point can be obtained by using this equation. Thus we can obtain the electromagnetic fields at $\mathrm{P}$ by applying (6) to the three components of electric and magnetic fields.

\subsection{Spreading of beams produced by image sources}

In order to investigate how the beams spread, their intensity profiles have been calculated for a plane at a distance $z$ from the image source. The image source given by (4) and (5) is now rotating around the $x$-axis by $\left(90^{\circ}-\alpha\right)$ so that the beam produced propagates along the $z$-axis (figure 2). The intensity profiles for the $\mathrm{TE}_{15}$ mode $\left(f=354 \mathrm{GHz}, f_{\mathrm{p}}=21.75 \mathrm{~mm}, a_{\mathrm{w}}=14 \mathrm{~mm}\right)$ are shown in figure 3 . Contours

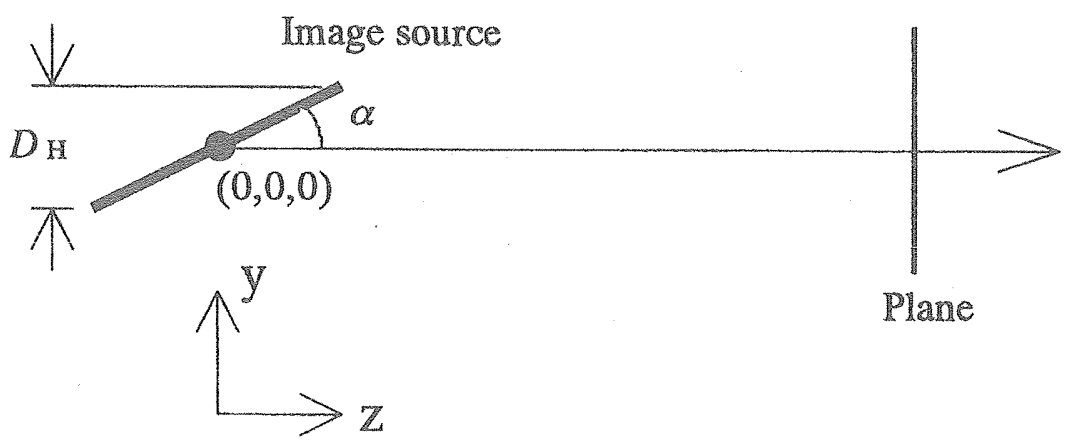

Figure 2. Plane image source used in the calculation of the subsequent radiation patterns of the quasi-optical antenna with a single parabolic reflector. The beam propagates along the $z$-axis. 
(a)

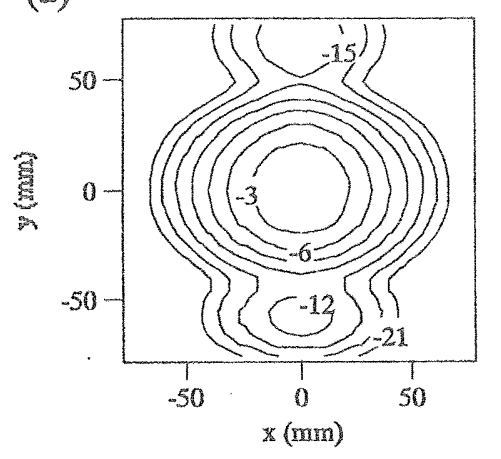

(c)

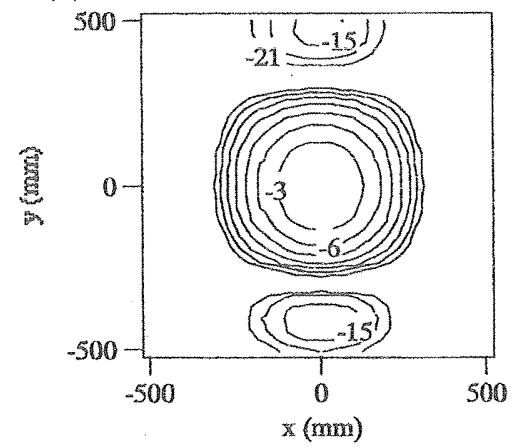

(b)

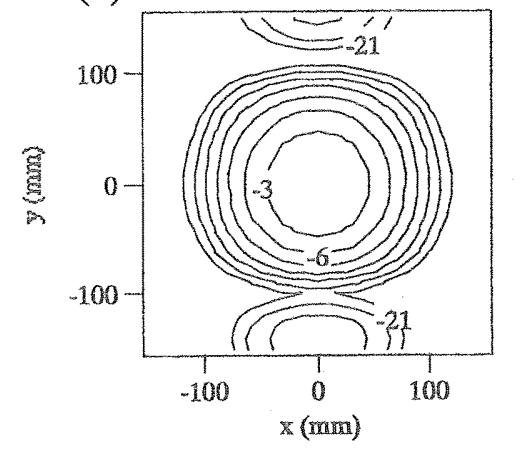

(d)

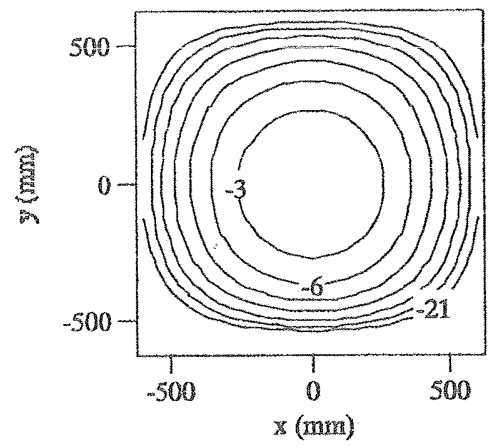

Figure 3. Calculated intensity contours at various distances $z$ from the image source: $(a)$ $z=3000 \mathrm{~mm} ;(b) z=7000 \mathrm{~mm} ;(c) z=20000 \mathrm{~mm} ;(d) z=40000 \mathrm{~mm}$. Contours are in decibels relative to the intensity maximum.

are in decibels relative to the intensity maximum. For this mode, the emission angle $\alpha$ is $8.23^{\circ}$ and the beam widths $D_{\mathrm{E}}$ and $D_{\mathrm{H}}$ and $87.0 \mathrm{~mm}$ and $55.4 \mathrm{~mm}$ respectively.

According to Gaussian optics, the beam intensity is given by

$$
I=\frac{w_{0 x} w_{0 y}}{w_{\mathrm{G} x} w_{\mathrm{G} y}} \exp \left(-\frac{2 x^{2}}{w_{\mathrm{G} x}^{2}}\right) \exp \left(-\frac{2 y^{2}}{w_{\mathrm{G} y}^{2}}\right)
$$

where $w_{0 x}$ and $w_{0 y}$ are the beam waist sizes, and $w_{\mathrm{G} x}$ and $w_{\mathrm{G} y}$ are the spot sizes defined by

$$
w_{G x}=w_{0 x}\left(1+\frac{\lambda^{2} z^{2}}{\pi^{2} w_{0 x}^{4}}\right)^{1 / 2}
$$

and

$$
w_{\mathrm{G} y}=w_{0 y}\left(1+\frac{\lambda^{2} z^{2}}{\pi^{2} w_{0 y}^{4}}\right)^{1 / 2}
$$

All of these sizes are the half-widths of the $-8.69 \mathrm{~dB}$ (or $\mathrm{e}^{-2}$ ) contours. Such a beam is described as bi-Gaussian. The beam spot sizes $w_{x}$ and $w_{y}$ are listed in table 1 


\begin{tabular}{lcccc}
\hline$z(\mathrm{~mm})$ & 3000 & 7000 & 20000 & 40000 \\
\hline$w_{x}(\mathrm{~mm})$ & 38.9 & 75.9 & 214 & 426 \\
$w_{x} / w_{\mathrm{Gx}}\left(w_{0 x}=25.4 \mathrm{~mm}\right)$ & 0.95 & 0.97 & 1.0 & 1.0 \\
$w_{y}(\mathrm{~mm})$ & 33.4 & 74.9 & 214 & 429 \\
$w_{y} / w_{\mathrm{G} y}\left(w_{0 y}=25.3 \mathrm{~mm}\right)$ & 0.82 & 0.95 & 1.0 & 1.0 \\
\hline
\end{tabular}

Table 1. Spot sizes of the beam produced by the image source for the $\mathrm{TE}_{15}$ mode $\left(f=354 \mathrm{GHz}, f_{\mathrm{p}}=21.75 \mathrm{~mm}, a_{\mathrm{w}}=14 \mathrm{~mm}\right)$ as a function of distance $z \cdot w_{\mathrm{G} x}$ and $w_{G y}$ denote spot sizes of a Gaussian beam with waist sizes of $w_{0 x}$ and $w_{0 y}$

as a function of distance $z$. The beam waist sizes calculated from the spot sizes at $z=40000 \mathrm{~mm}$ using (8) and (9) are $w_{0 x}=25.4 \mathrm{~mm}$ and $w_{0 y}=25.3 \mathrm{~mm}$, respectively. The ratios $w_{x} / w_{\mathrm{G} x}$ and $w_{y} / w_{\mathrm{G} y}$ demonstrate that the spot size for larger $z$ are accurately given by Gaussian optics. This is expected to be the case in the far field where $z \gg z_{\mathrm{F}}$, given by

$$
z_{F}=\frac{1}{4} k\left(D_{\mathrm{E}}^{2}+D_{\mathrm{H}}^{2}\right)
$$

In the present case, the value of $z_{F}$ is $19700 \mathrm{~mm}$.

The waist sizes of the equivalent bi-Gaussian beam are obtained for image sources at various gyrotron operating wavelengths (table 2). The waist size $w_{0 y}$ depends mainly on the only length $D_{\mathrm{H}}$ and the waist size $w_{0 x}$ depends mainly on the only width $D_{\mathrm{E}}$. The ratio $w_{0 y} / D_{\mathrm{H}}$ is approximately equal to 0.46 and the ratio $w_{0 x} / D_{\mathrm{E}}$ is approximately equal to 0.44 for $\mathrm{TE}_{0 \mathrm{n}}$ modes and 0.30 for $\mathrm{TE}_{\mathrm{ln}}$ modes. These results can be used as the starting point for the design of a quasi-optical antenna which can produce a far field with circular cross-section. We will show this for the $T E_{15}$ mode case where $D_{\mathrm{E}}=87.0 \mathrm{~mm}$ and $D_{\mathrm{H}}=55.4 \mathrm{~mm}$. We will treat the beam as Gaussian with a waist size of $w_{0}=25.4 \mathrm{~mm}$ because its crosssection is almost circular $\left(w_{0 x}=25.4 \mathrm{~mm}, w_{0 y}=25.3 \mathrm{~mm}\right)$, and use the complex beam parameter. This will lead to a method of designing systems that requires much less computation time.

\begin{tabular}{|c|c|c|c|c|c|c|c|}
\hline Mode & $\underset{(\mathrm{GHz})}{f}$ & $\begin{array}{c}\alpha \\
\left({ }^{\circ}\right)\end{array}$ & $\begin{array}{c}z_{\mathrm{F}} \\
(\mathrm{mm})\end{array}$ & $\begin{array}{c}D_{\mathrm{E}} \\
(\mathrm{mm})\end{array}$ & $w_{0 x} / D_{\mathrm{E}}$ & $\begin{array}{c}D_{\mathrm{KI}} \\
(\mathrm{mm})\end{array}$ & $w_{0 y} / D_{\mathrm{H}}$ \\
\hline $\mathrm{TE}_{03}$ & 301 & 6.62 & $\begin{array}{l}10500 \\
16800 \\
20600\end{array}$ & $\begin{array}{r}60.0 \\
87.0 \\
100.0\end{array}$ & $\begin{array}{l}0.43 \\
0.44 \\
0.44\end{array}$ & $\begin{array}{l}55.6 \\
55.6 \\
55.6\end{array}$ & $\begin{array}{l}0.46 \\
0.45 \\
0.46\end{array}$ \\
\hline $\mathrm{TE}_{06}$ & 388 & 9.93 & $\begin{array}{ll}13 & 500 \\
21 & 600 \\
26 & 500\end{array}$ & $\begin{array}{r}60.0 \\
87.0 \\
100.0\end{array}$ & $\begin{array}{l}0.43 \\
0.44 \\
0.44\end{array}$ & $\begin{array}{l}55.2 \\
55.2 \\
55.2\end{array}$ & $\begin{array}{l}0.46 \\
0.46 \\
0.46\end{array}$ \\
\hline $\mathrm{TE}_{15}$ & 354 & 8.23 & $\begin{array}{l}12400 \\
19700 \\
24200\end{array}$ & $\begin{array}{r}60.0 \\
87.0 \\
100.0\end{array}$ & $\begin{array}{l}0.30 \\
0.29 \\
0.30\end{array}$ & $\begin{array}{l}55.4 \\
55.4 \\
55.4\end{array}$ & $\begin{array}{l}0.46 \\
0.46 \\
0.46\end{array}$ \\
\hline $\mathrm{TE}_{18}$ & 718 & 9.30 & $\begin{array}{l}13100 \\
19400 \\
34300\end{array}$ & $\begin{array}{l}44.0 \\
60.0 \\
87.0\end{array}$ & $\begin{array}{l}0.29 \\
0.30 \\
0.30\end{array}$ & $\begin{array}{l}39.5 \\
39.5 \\
39.5\end{array}$ & $\begin{array}{l}0.46 \\
0.46 \\
0.46\end{array}$ \\
\hline
\end{tabular}

Table 2. Waist sizes of equivalent bi-Gaussian beam obtained for various image sources. These sizes are compared with beam widths $D_{\mathrm{E}}$ and $D_{\mathrm{H}}$. 
3. Cstimation of the position and size of beam waist by using the connplex bexm parameter

\subsection{Description of the complex beam parameter}

A Gaussian beam with waist size $w_{0}$ has a wave front with a radius of curvature given by

$$
R=z\left(1+\frac{\pi^{2} w_{0}^{4}}{\lambda^{2} z^{2}}\right)
$$

The complex beam parameter is defined by

$$
\frac{1}{q}=\frac{1}{R}-j \frac{\lambda}{\pi w^{2}}
$$

Because $R=\infty$ at the beam waist, the complex beam parameter there is

$$
q_{0}=j \frac{\pi w_{0}^{2}}{\lambda}
$$

The complex beam parameter varies along the beam propagation and can be changed by focusing the beam with a thin lens. The complex beam parameter, after propagating a distance $d$, changes to

$$
q^{\prime}=q+d
$$

After the lens, it changes to a new value given by

$$
\frac{1}{q^{\prime}}=\frac{1}{q}-\frac{1}{f}
$$

where $f$ denotes the focal length of the lens.

\subsection{Elliptical mirror}

An elliptical mirror can be treated as a simple focusing element with an equivalent focal length $f$ given by

$$
\frac{1}{f}=\frac{1}{d_{1}}+\frac{1}{d_{2}}
$$

where $d_{1}$ and $d_{2}$ are respectively the distance between focal points and the centre of the cut section of the elliptical surface (figure 4). Although its shape is determined by the incidence angle $\theta$ and distances $d_{1}$ and $d_{2}$, its function is determined only by the focal length $f$. This result is tested numerically in $\$ 4$.

\subsection{Application of the complex beam parameter}

As an illustration of the model, we will design a quasi-optical transmission line (see figure 5) to produce a well collimated beam with a waist size of $w_{0}^{\prime}=5.0 \mathrm{~mm}$.

We first calculate the complex beam parameter $q_{0}$ at the image source, using (13) with $w_{0}=25.4 \mathrm{~mm}$. Next we calculate $q$ at the elliptical mirror, located at a distance $d$ further away, using (14). Next we calculate $q^{\prime}$ after reflection by the elliptical mirror of focal length $f$, using (15). We have two parameters that we can adjust, 


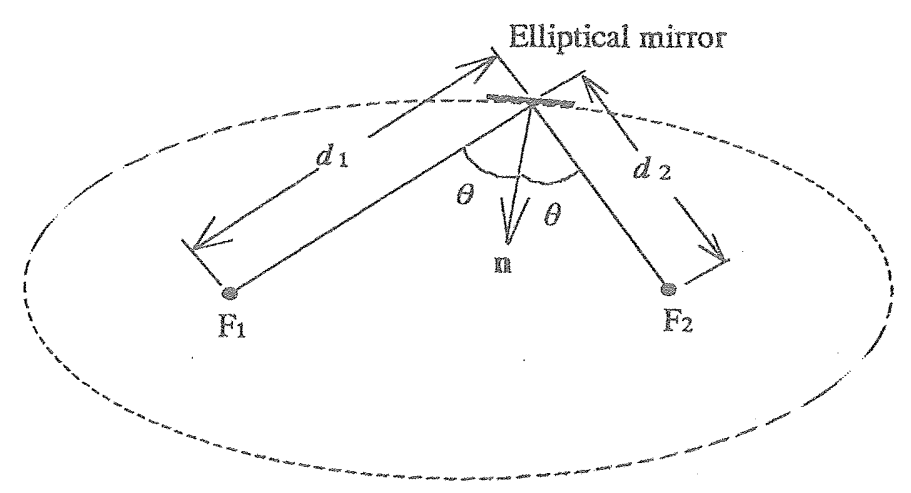

Figure 4. Geometry of elliptical mirror.

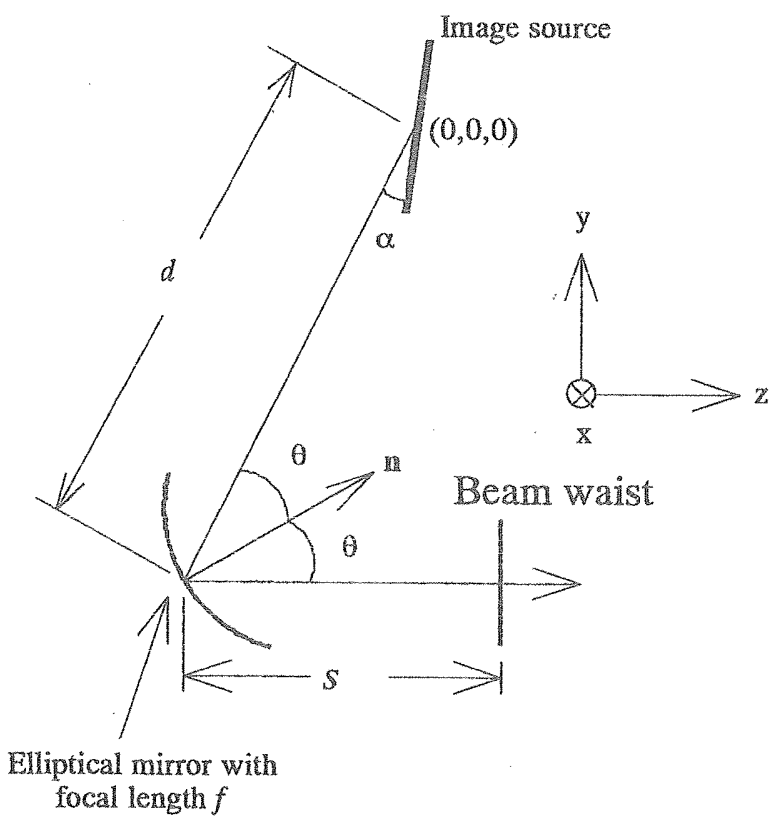

Figure 5. Quasi-optical transmission line.

namely $d$ and $f$, in order to give the desired beam waist $w_{0}^{\prime}=5.0 \mathrm{~mm}$ at the required position $s=1453 \mathrm{~mm}$. In this case $d=7000 \mathrm{~mm}, f=1230 \mathrm{~mm}$.

\section{Verification of the concept of focal length to wave optics}

It is important to see to what extent the reflected beam is infiuenced by the incident angle $\theta$ and the distances $d_{1}, d_{2}$ (see figure 4). Calculations using the Huygens equation have been carried out for $f=1230 \mathrm{~mm}$ and a mirror of length $W_{\mathrm{h}} / \cos \theta$ and width $240 \mathrm{~mm}$. This mirror projects a rectangle of height $W_{\mathrm{h}}$ and width $240 \mathrm{~mm}$ on the $x-y$ plane (figure 5). The dependence of the incident angle is shown in figure 6 and the dependence on the combination of $d_{1}$ and $d_{2}$ is shown in figure 7 . These results prove that incident angle and the combination have little effect on the beams, and support the use of the concept of focal length in wave optics. 
(a)

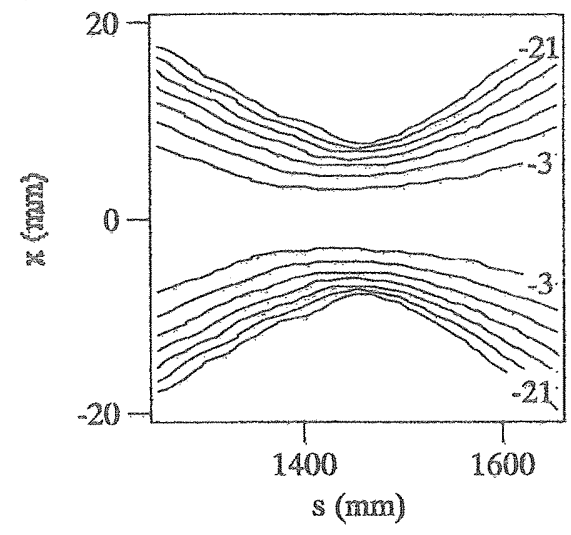

(c)

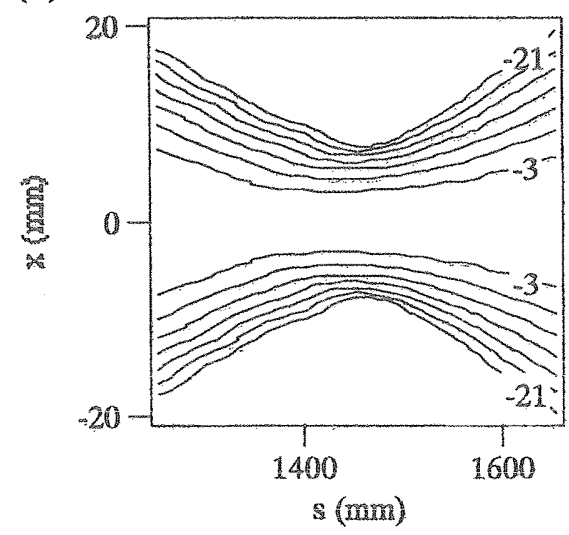

(e)

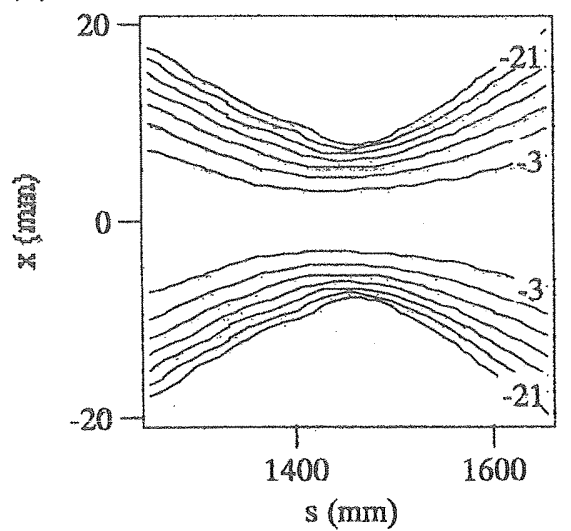

(b)

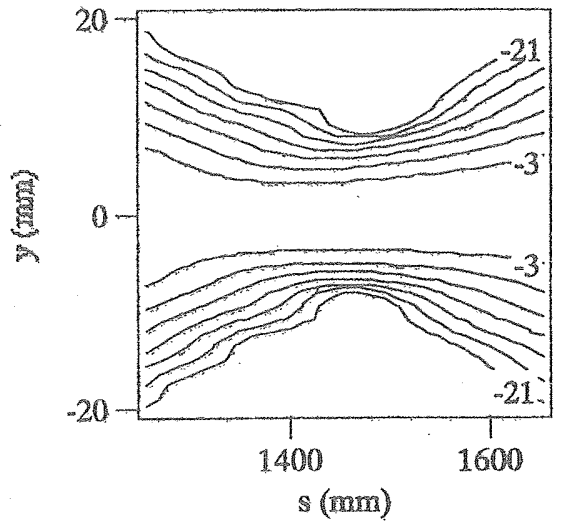

(d)

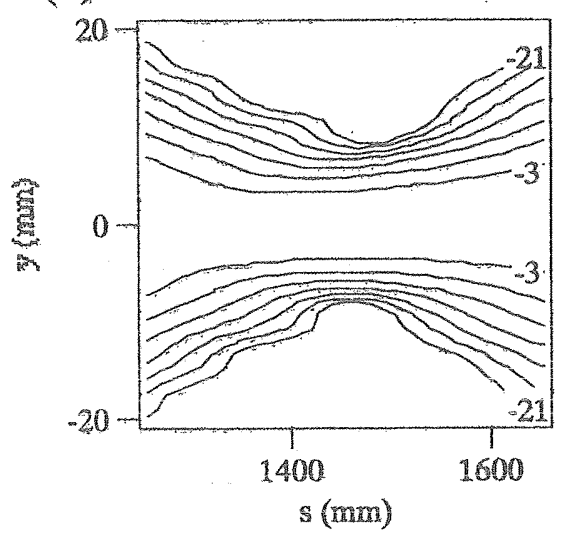

(f)

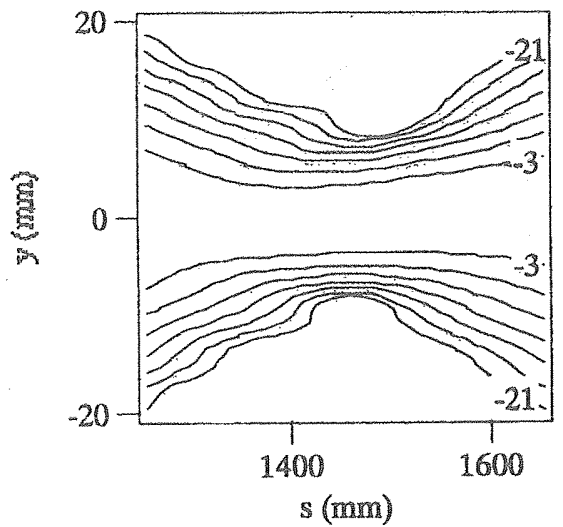

Figure 6. Calculated intensity contours in the $x-s$ plane and the $y-s$ plane. Contours are relative to the intensity along the $s$-axis. Calculations are carried out under the conditions of $d_{1}=7000 \mathrm{~mm}, d_{2}=1492 \mathrm{~mm}$ and $W_{\mathrm{h}}=210 \mathrm{~mm} .(a),(b) \theta=0^{\circ} ;(c),(d)$ $\theta=22.5^{\circ} ;(e),(f) \theta=45^{\circ}$. 
(a)

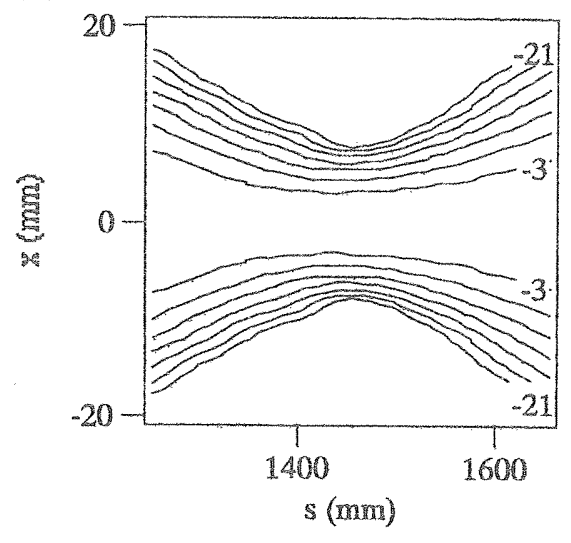

(c)

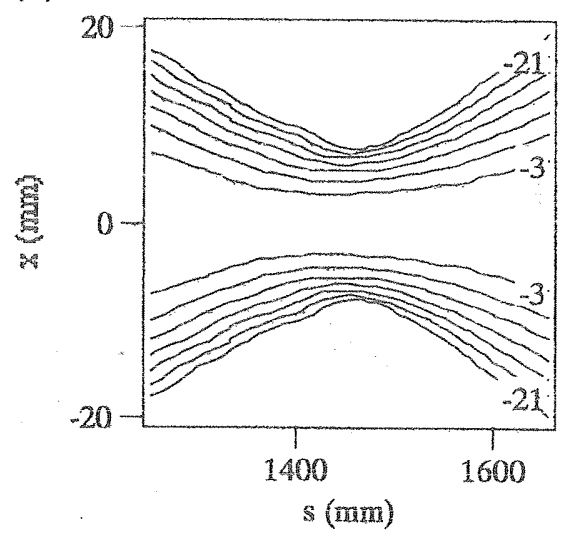

(e)

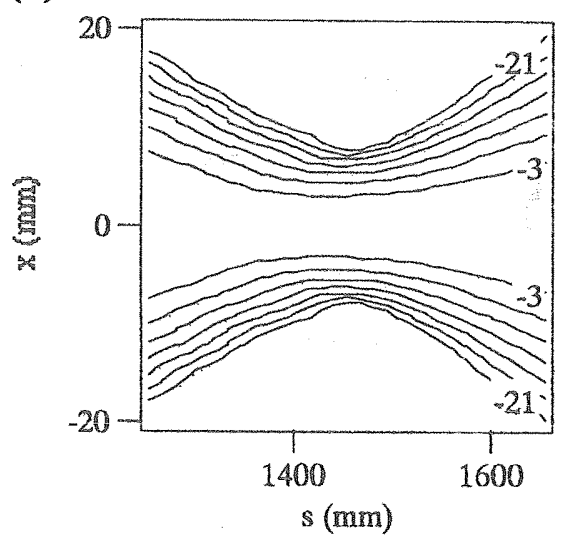

(b)

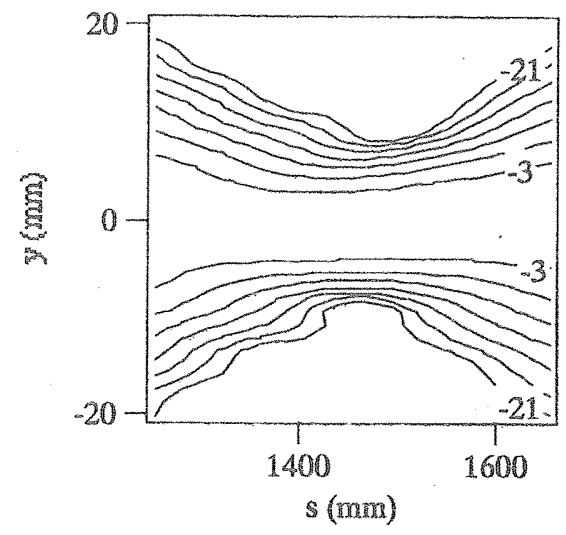

(d)

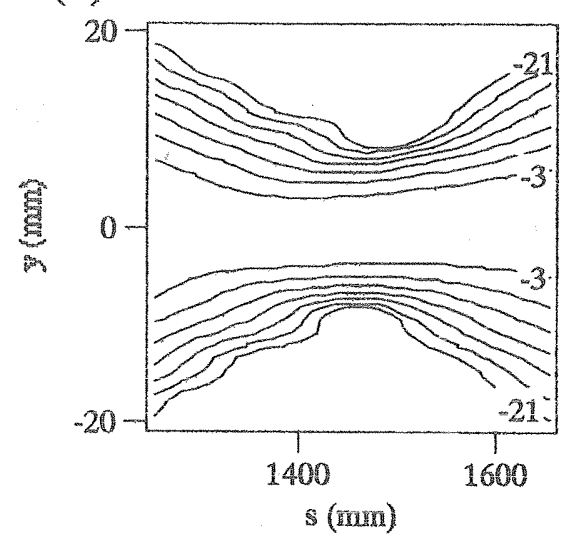

(f)

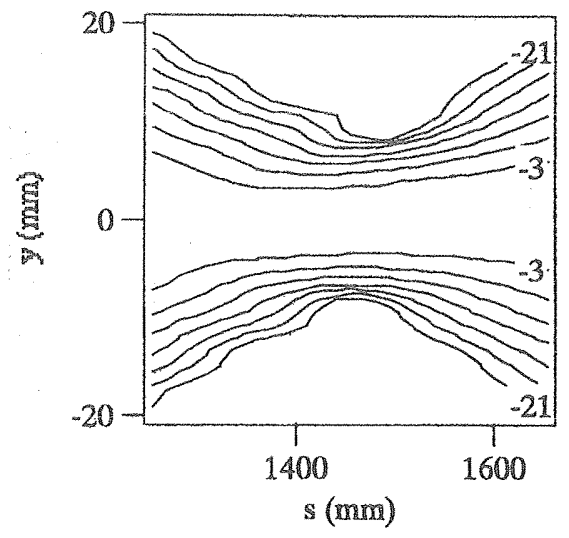

Figure 7. Calculated intensity contours in the $x-s$ plane and the $y-s$ plane. Contours are relative to the intensity along the $s$-axis. Calculations are carried out for $\theta=45^{\circ}$ and $W_{\mathrm{h}}=210 \mathrm{~mm} .(a),(b) d_{1}=5000 \mathrm{~mm}, d_{2}=1631 \mathrm{~mm} ;(c),(d) d_{1}=7000, d_{2}=1492 \mathrm{~mm}$; (e), $(f) d_{1}=10000 \mathrm{~mm}, d_{2}=1402 \mathrm{~mm}$. 


\section{Hofluence of the elliptical minror size on the beam shape}

The beam produced by the image source contains sidelobes in addition to the main beam, as can be seen in figure $3(c)$. The quality of the beam is improved by truncating the sidelobes, which is done by limiting the size of the elliptical mirror. Calculated intensity contours at the beam waist for different mirror sizes $W_{\mathrm{h}}$ are shown in figure 8. A wider mirror distorts the beam because there are contributions from the sidelobes (figure $8(c)$ and $(d)$ ). On the other hand, a smaller mirror truncates the main beam and produces an elongated beam (figure $8(a)$ ). The optimum mirror size which truncates the sidelobes but does not add any distortion of its own is shown in figure $8(b)$. In spite of the truncation of the sidelobes, most of the power from the image source $(89.3 \%)$ is still reflected by the mirror.

(a)

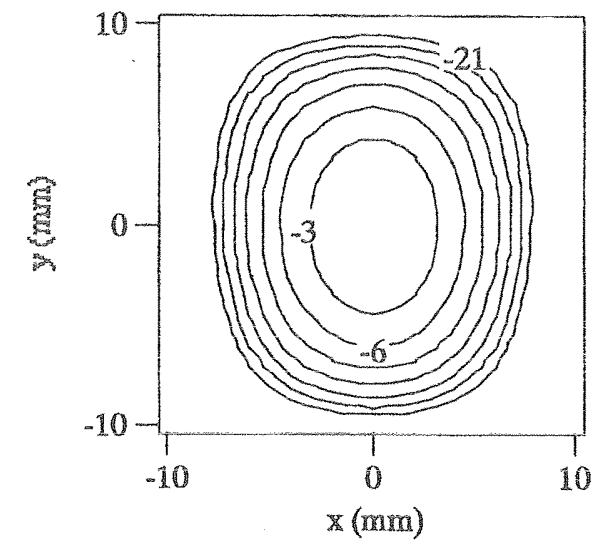

(c)

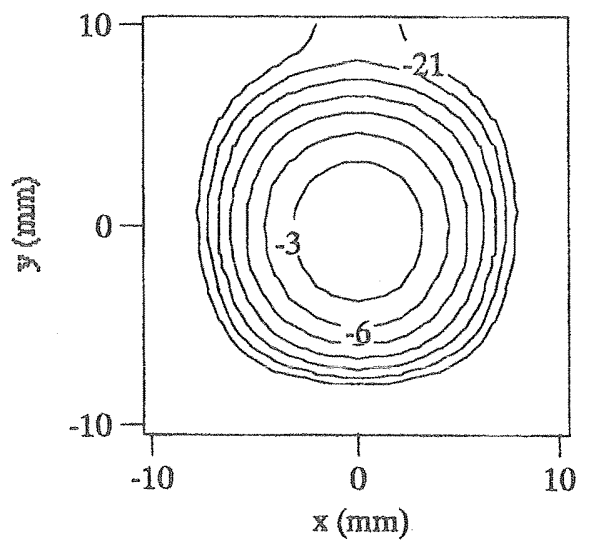

(b)

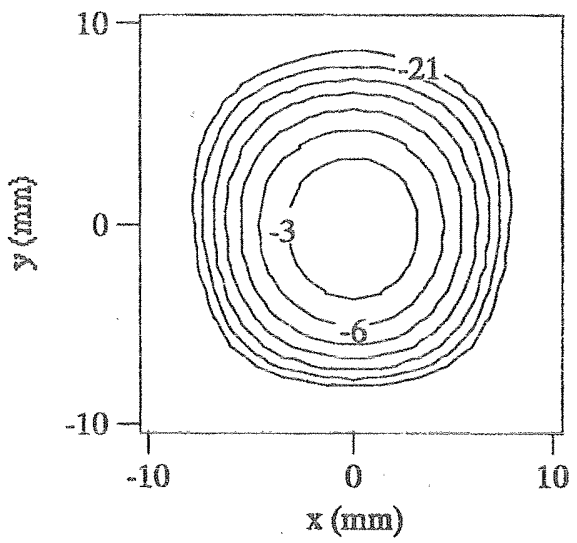

(d)

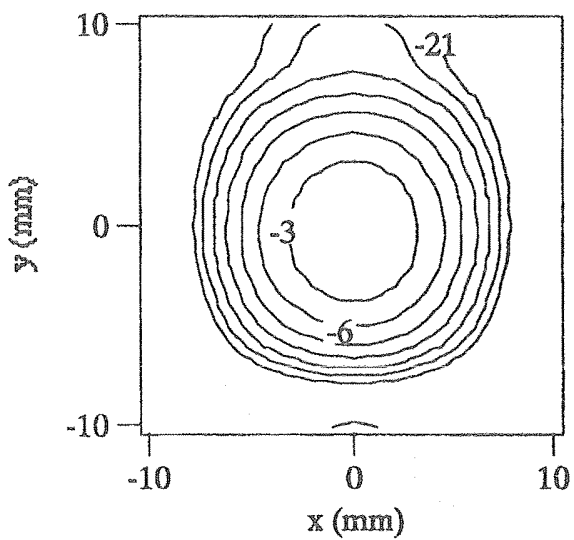

Figure 8. Calculated intensity contours at the beam waist predicted by the beam parameter $(s=1453 \mathrm{~mm})$. Calculations are carried out for $d_{1}=7000 \mathrm{~mm}, d_{2}=1492 \mathrm{~mm}, \theta=45^{\circ}$. (a) $W_{\mathrm{h}}=141 \mathrm{~mm} ;(b) W_{\mathrm{h}}=210 \mathrm{~mm} ;(c) W_{\mathrm{h}}=248 \mathrm{~m} ;(d) W_{\mathrm{h}}=283 \mathrm{~mm}$. 


\section{Verification of estimation hy the complex beam parameter}

The complex beam parameter has been applied to the design of systems, producing beams with different waist sizes of $w_{0}^{\prime}=2.0 \mathrm{~mm}, 10.0 \mathrm{~mm}$ and $15.0 \mathrm{~mm}$ as well as $w_{0}^{\prime}=5.0 \mathrm{~mm}$. Calculated intensity contours are shown in figure 9 . The corresponding

(a)

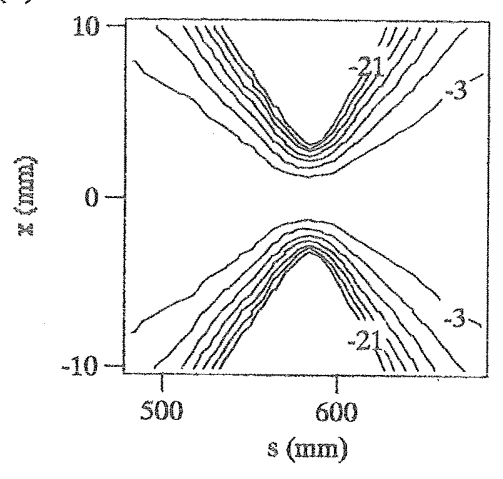

(c)

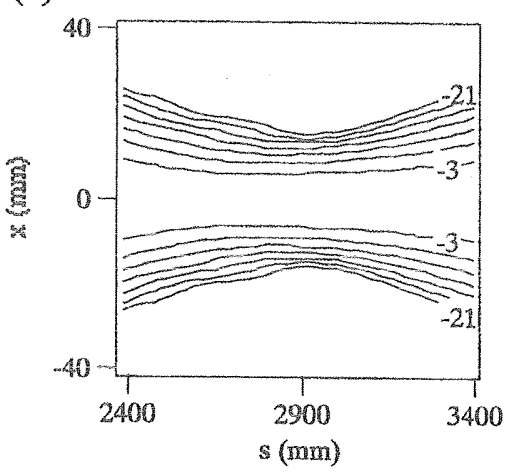

(e)

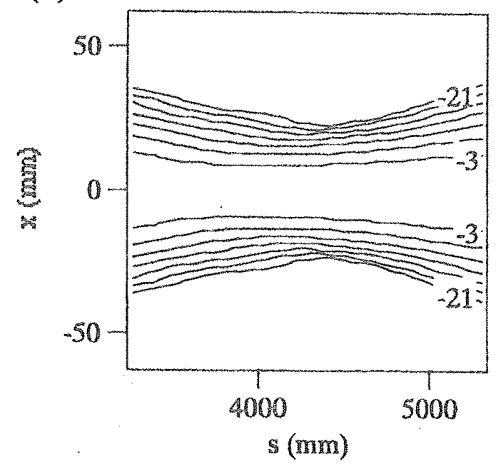

(b)

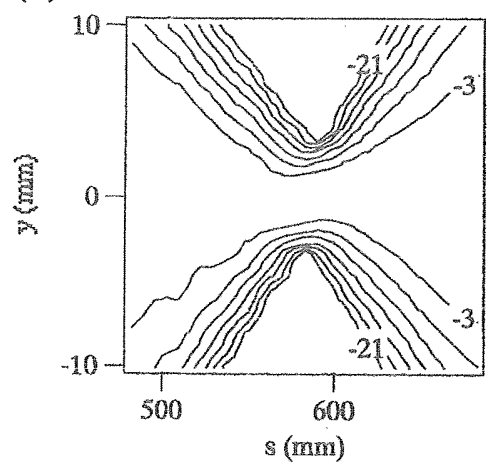

(d)

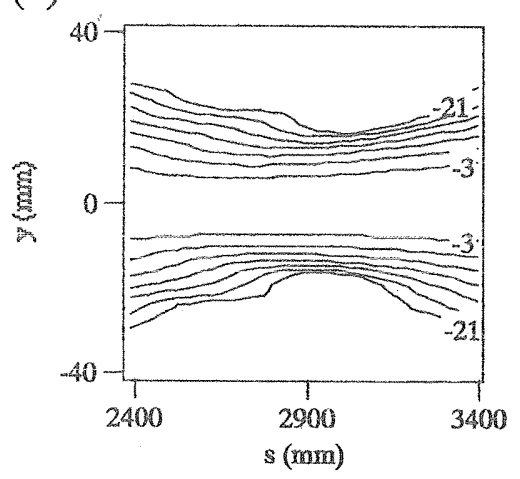

(f)

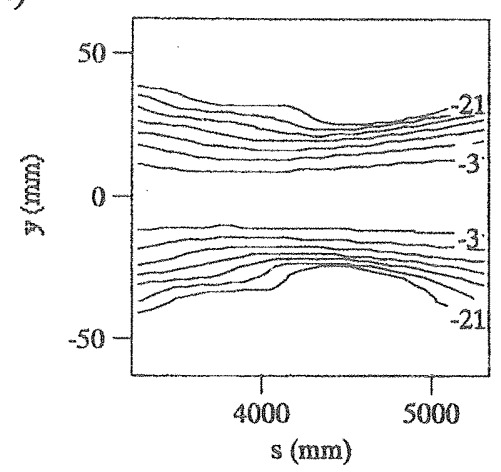

Figure 9. Calculated intensity contours in the $x-s$ plane and the $y-s$ plane. Contours are relative to the intensity along the $s$-axis. Calculations are carried out for $\theta=45^{\circ}$, $W_{\mathrm{h}}=210 \mathrm{~mm}$ and $d_{1}=7000 \mathrm{~mm} .(a),(b) \quad w_{0}^{\prime}=2.0 \mathrm{~mm}, d_{2}=588 \mathrm{~mm} ;(c),(d)$ $w_{0}^{\prime}=10.0 \mathrm{~mm}, d_{2}=3071 \mathrm{~mm} ;(e),(f) w_{0}^{\prime}=15.0 \mathrm{~mm}, d_{2}=4767 \mathrm{~mm}$. $w_{0}^{\prime}$ denotes the objective waist size. 
focal lengths $f$ and the distances $s$ from the elliptical mirror to the beam waist (see figure 5) are listed in table 3. The waist sizes $w_{0 x}^{\prime}$ and $w_{0 y}^{\prime}$ and its distances $s_{x}$ and $s_{y}$ obtained by a Huygens equation calculation are also listed in table 3, for comparison. The results obtained by the complex beam parameter approach are in good agreement with the calculations. Calculated intensity contours at the beam waist predicted by the complex beam parameter are shown in figure 10 .

\begin{tabular}{lrrrrrr}
\hline $\begin{array}{l}w_{0}^{\prime} \\
(\mathrm{mm})\end{array}$ & $\begin{array}{c}f \\
(\mathrm{~mm})\end{array}$ & $\begin{array}{c}s \\
(\mathrm{~mm})\end{array}$ & $\begin{array}{c}w_{0 x}^{\prime} \\
(\mathrm{mm})\end{array}$ & $\begin{array}{c}s_{x} \\
(\mathrm{~mm})\end{array}$ & $\begin{array}{c}w_{0 y}^{\prime} \\
(\mathrm{mm})\end{array}$ & $\begin{array}{r}s_{y} \\
(\mathrm{~mm})\end{array}$ \\
\hline 2.0 & 542 & 582 & 2.1 & 583 & 2.3 & 584 \\
5.0 & 1230 & 1453 & 5.3 & 1450 & 5.7 & 1450 \\
10.0 & 2134 & 2889 & 10.6 & 2828 & 11.4 & 2811 \\
15.0 & 2836 & 4288 & 15.5 & 414.3 & 17.0 & 4049 \\
\hline
\end{tabular}

Table 3. Waist sizes $w_{0}^{\prime}$ and distances $s$ from the elliptical mirror to the beam waist. The value of $w_{0}^{\prime}$ is a target waist size of the beam produced. That of $f$ denotes the focal length determined by the complex beam parameter. The values of $w_{0 x}^{\prime}$ and $w_{0 y}^{\prime}$ are waist sizes obtained by calculations.

(a)

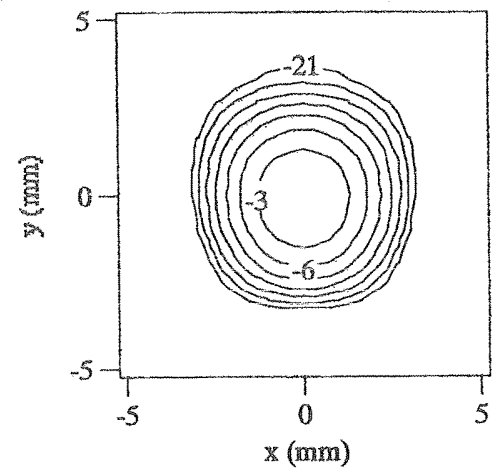

(c)

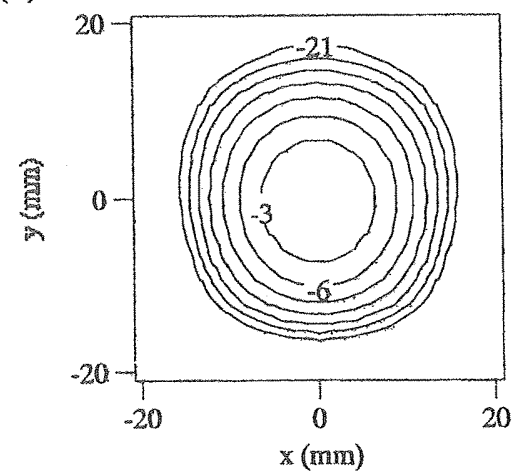

(b)

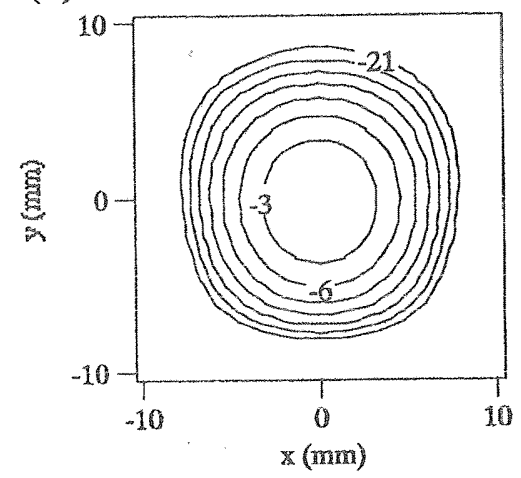

(d)

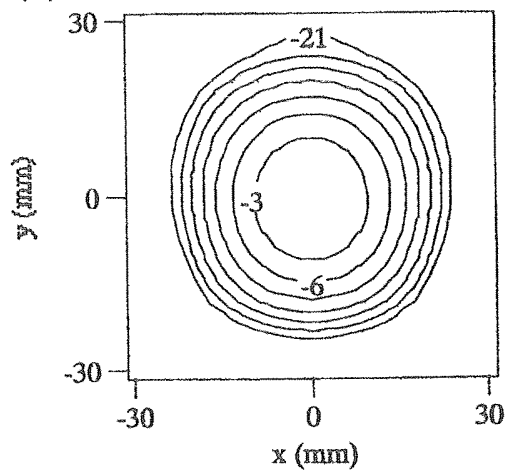

Figure 10. Calculated intensity contours at the beam waist predicted by the beam parameter. Calculations are carried out for $\theta=45^{\circ}, W_{\mathrm{h}}=210 \mathrm{~mm}$ and $d_{1}=7000 \mathrm{~mm}$. (a) $w_{0}^{\prime}=2.0 \mathrm{~mm}, \quad d_{2}=588 \mathrm{~mm}, \quad s=482 \mathrm{~mm} ; \quad(b) \quad w_{0}^{\prime}=5.0 \mathrm{~mm}, d_{2}=1492 \mathrm{~mm}$, $s=1453 \mathrm{~mm} ;(c) w_{0}^{\prime}=10.0 \mathrm{~mm}, d_{2}=3071 \mathrm{~mm}, s=2889 \mathrm{~mm} ;(d) w_{0}^{\prime}=15.0 \mathrm{~mm}$, $d_{2}=4767 \mathrm{~mm}, s=4288 \mathrm{~mm}$. $w_{0}^{\prime}$ denotes the objective waist size. 


\section{Conclusion}

The quasimoptical transmission line described here is able to convert the gyrotron output into a well collimated, linearly polarized beam. For the sake of beam quality, non-paraxial components and sidelobes of the beam produced by the quasi-optical antenna are removed by locating an elliptical mirror of suitable size far from the antenna. Very little $(<11 \%)$ of the beam power is lost.

Calculations using the Huygens equation confirm that the propagation of the beam produced by the image source can be treated by Gaussian optics far from the source and that the usual geometrical optics concept of focal length is useful here. This allows us to apply the complex beam parameter to the design of transmission lines to produce beams with the spot size we require. Using this approach requires much less computation time.

\section{Acknowledgunents}

The authors would like to thank Dr G. F. Brand, University of Sydney, for valuable discussions and help.

This work was done as a collaboration between Fukui University, the National Institute for Fusion Science and Universität Stuttgart, Germany. It has been supported by the Japan Society for the Promotion of Science (International Joint Research Projects).

The work in Fukui University was partially supported by a Grant-in-Aid from the Ministry of Education, Science and Culture of Japan.

Numerical calculations were made at the National Institute for Fusion Science Computer Center.

\section{Relerences}

Brand, G. F., Fekete, P. W. Idehara, T., and Moore, K. J., 1990, Quasi-optical antennas for plasma scattering. International Joumal of Electronics, 68, 1063-1073.

Fekete, P.W., Brand, G. F, and IDEhara, T., 1994, Scattering from discrete Alfven waves in a tokamak using a gyrotron radiation source. Plasma Physics and Controlled Nuclear Fusion, 36, 1407-1417.

Hong, K. D., Brand, G. F., Fekete, P. W., and Idekara, T., 1992, Submillimeter wave generation by second harmonic operation of tunable gyrotrons. International Journal of Infrared and Millimeter Waves, 13, 215-227.

Iofmara, T., Tatsukawa, T., Ogawa, I., Tanabe, H., Mori, T., Wada, S., Brand, G. F., and BRENNAN, M. H., 1992, Development of a second cyclotron harmonic gyrotron operating at submillimeter wavelengths. Physics of Fluids, $\mathbb{R} 4$, 267-273.

Idemara, T., Tatsukawa, T., Ogawa, I., Shmizu, Y, Makino, S., and Kanemaki, T., 1993, Development of a high-frequency, second-harmonic gyrotron tunable up to $636 \mathrm{GHz}$. Physics of Fluids, $135,1377-1379$.

KaWahata, K., Tetsuka, T, Fumta, J, NaGatsu, M., OHnishi, H., OKamma, S., and IWEASAKY, T., 1988, HCN laser scattering on the JPP T-IIU tokamak. International Journal of Infrared and Millimeter Waves, 9, 655-665.

Ogawa, I., Sakai, A., Idemara, T., Kawahata, K., and Kasparek, W., 1997, A quasi-optical transmission line for plasma scattering measurements using a submillimeter wave gyrotron. International Journal of Electronics, 83, 635-644.

Semet, A., Mase, A., Peebles, W. A., Luhmann, N. C., Zweben, S., Jr, 1980, Study of lowfrequency microturbulence in the Microtor tokamak by far-infrared laser scattering. Physical Review Letters, $45,445-448$. 
Suvorov, E. V., Holzhauer, E., Kasparek, W., Lubyako, L. V., Burov, A. B., Dryagin, Y. A., Filchenkov, S. E., Fraiman, A. A., Kukin, L. M., Kostrov, A. V., Ryndyk, D. A., Shtanyuk, A. M., Skalyga, N. K., Smolyakova, O. B., Erckmann, V., Geist, T., Kick, M., Laqua, H., and Rust, M., 1997, Collective Thomson scattering at WA-AS. Plasma Physics and Controlled Fusion, 39, B337-B351.

Vlasov, S. N., and ORLOVA, I. M., 1974, Quasioptical transformer which transforms the waves in a waveguide having a circular cross section into a highly directional wave beam. Radiofizika, 17, 115-119.

WADA, O., and NAKAJIMA, M., 1986, Reflector antennas for electron cyclotron resonance heating of fusion plasma. Space Power, 6, 213-220. 